\title{
$\bar{s}$ Striction Lines of Non-developable Ruled Surfaces in Euclidean 3-Space*
}

\author{
Ali ÇAKMAK
}

\begin{abstract}
The ruled surfaces, one of the areas of interest of differential geometry, have been one of the surface types studied by many mathematicians from the past to the present day. Similarly, some special curves which helix, slant helix, Bertrand curve, etc. are also the curve types discussed often by mathematicians. In this paper, it will be shown that striction lines of non-developable ruled surfaces are helix, slant helix, Bertrand or Mannheim curve in some special cases.
\end{abstract}

Keywords: Non-developable ruled surface, line of striction, helix, Bertrand curve, Mannheim curve.

\section{Öklid-3 Uzayında Açılabilir Olmayan Regle Yüzeylerin Striksiyon Çizgileri}

ÖZET: Diferansiyel geometrinin ilgi alanlarından olan regle yüzeyler, geçmişten günümüze bir çok matematikçi tarafından çalışılan yüzey tiplerinden birisidir. Benzer şekilde helis, slant helis, Bertrand eğrisi gibi bazı özel eğriler de matematikçiler tarafından sıklıkla tartışılan eğri tipleridir. Bu makalede, bazı özel durumlarda açılabilir olmayan regle yüzeylerin striksiyon çizgilerinin helis, slant helis, Bertrand eğrisi ya da Mannheim eğrisi olduğu gösterilecektir.

Anahtar Kelimeler: Açılabilir olmayan regle yüzey, striksiyon çizgisi, helis, Bertrand eğrisi, Mannheim eğrisi.

Ali ÇAKMAK (0000-0002-2783-9311), Bitlis Eren Üniversitesi, Fen Edebiyat Fak., Matematik, Bitlis, Türkiye Sorumlu yazar/Corresponding Author: Ali ÇAKMAK, acakmak@beu.edu.tr

Bu makale 22-24 Ağustos 2017 tarihinde Van ilinde Caucasian Mathematics Conference II (CMC - II)'de sunulmuştur. 


\section{INTRODUCTION}

It is known that if a surface is formed by the movement of a line, this surface is called the ruled surface. A ruled surface is the locus of a line depending on a parameter. We assume that this line does not have an envelope; hence the surface is

parameters $u$ and $v$ in $E^{3}$ by $X(u, v)$. Let

$X(u, v)=a(u)+v b(u)$

be a non-developable ruled surface in $E^{3}$ with $b^{2}(u)=1$ and the parameter $u$ is the arc length parameter of $b(u)$ as a unit spherical curve in $E^{3}$ (Liu et al., 2014; Yu et al., 2014). If $a^{\prime}(u) \cdot b^{\prime}(u)=0$, base curve $a(u)$ is striction line of ruled surface.

\section{MATERIAL AND METHOD}

Definition 2.1. A curve $\beta$ with $\kappa(s) \neq 0$ is defined as a cylindrical helix if the tangent lines of the curve

$$
\left(\frac{\tau}{\kappa}\right)(s)=\text { constant }
$$

Here, if $\kappa(s)$ and $\tau(s)$ are constants we call a circular helix.

Definition 2.2. A curve $\beta$ with $\kappa(s) \neq 0$ is non-developable (Kühnel, 2006). In other words, it is mean that a non-developable surface free of points of vanishing Gaussian curvature in a 3-dimensional Euclidean space (Yoon, 2007). We denote the Euclidean 3-space by $E^{3}$ and a regular parameter surface with the

Some special curves which are helix, slant helix, Bertrand and Mannheim curves are examined by the some authors. (Izumiya and Takeuchi, 2002; Liu and Wang, 2008; Yaylı and Saraçoğlu, 2012).

$\beta$ creative a constant angle with a fixed direction. Proposition 2.1. A curve $\beta$ with $\kappa(s) \neq 0$ is a cylindrical helix if and only if

defined as a slant helix if the principal normal lines of $\beta$ creative a constant angle with a fixed direction. 
Proposition 2.2. Let $\beta$ be an unit speed space curve with $\kappa(s) \neq 0$. Then, $\beta$ is a slant helix if and only if

$$
\sigma(s)=\left(\frac{\kappa^{2}}{\left(\kappa^{2}+\tau^{2}\right)^{\frac{3}{2}}}\left(\frac{\tau}{\kappa}\right)^{\prime}\right)(s)
$$

is a constant function.

Definition 2.3. A curve $\beta: I \rightarrow E^{3}$ with $\kappa(s) \neq 0$ is defined as a Bertrand curve if there is a curve $\bar{\beta}: I \rightarrow E^{3}$ under the condition that the principal

$A\left(\tau^{\prime}(s) \kappa(s)-\kappa^{\prime}(s) \tau(s)\right)-\tau^{\prime}(s)=0$

such that $A \neq 0$ and $A \in R$. Here, $\bar{\beta}=\beta(s)+A n(s)$ is Bertrand mate of $\beta$.

Definition 2.4. A curve $\beta: I \rightarrow E^{3}$ with $\kappa(s) \neq 0$ is defined as a Mannheim curve if there is a curve $\bar{\beta}: I \rightarrow E^{3}$ such that the principal normal lines

$\kappa=c\left(\kappa^{2}+\tau^{2}\right)$,

where $c$ is a nonzero constant and $\kappa$ and $\tau$ are curvature and torsion of curve, respectively.

Definition 2.5. A ruled surface is defined by transformation

$X(a, b): I x E \rightarrow E^{3}$

$(u, v) \rightarrow X(a, b)(u, v)=a(u)+v b(u)$, 
where $a: I \rightarrow E^{3}, b: I \rightarrow E^{3}$ are differentiable transformations and $I$ is an open interval. $a(u)$ and $b(u)$ are called base curve and director curve, respectively. If $b(u)$ is a constant, ruled surface is called as a cylinder.

It is known that if Gaussian curvature is zero, the ruled surface is developable ruled surface. In addition, it is mean that a non-developable ruled surface free of points of vanishing Gaussian curvature.

$$
\begin{aligned}
& x^{\prime}(u)=\alpha(u), \\
& \alpha^{\prime}(u)=-x(u)+k_{g}(u) y(u), \\
& y^{\prime}(u)=-k_{g}(u) \alpha(u),
\end{aligned}
$$

where $k_{g}(u)$ is called the spherical curvature function and $\{x(u), \alpha(u), y(u)\}$ is called the spherical Frenet frame of the spherical curve $b(u)$ (Liu et al., 2014; Yu et al., 2014).

Definition 2.7. Suppose that $X(u, v)=a(u)+v b(u)$ is a non-developable ruled surface and $a(u)$ is the striction line of $X(u, v)$ under the conditions $a^{\prime}(u)=\lambda(u) x(u)+\mu(u) y(u)$ and $u$ is the arc length parameter of $b(u)$. Let $k_{g}(u)$ be the spherical curvature function of $b(u)$, then $X(u, v)$ can be given by $\left\{k_{g}(u), \lambda(u), \mu(u)\right\}$ up to a transformation in $E^{3}$. Here, the functions $k_{g}(u)$,
Definition 2.6. Suppose that $X(u, v)=a(u)+v b(u)$ is a non-developable ruled surface in $E^{3}$. We assume that $b(u)$ is a unit spherical curve in $E^{3}$ such that $b^{2}(u)=1$ and the parameter $u$ is the arc length parameter of $b(u)$ and base curve $a(u)$ is the striction line of the non-developable ruled surface $X(u, v)$. In this case, if we write as $x(u)=b(u), x^{\prime}(u)=\alpha(u)$ and $y(u)=\alpha(u)^{\prime} x(u)$, the spherical Frenet formulas of the curve $b(u)$ can be given by

$\lambda(u)$ and $\mu(u)$ are defined as structure functions of $X(u, v)$ in $E^{3}$ (Liu et al., 2014; Yu et al., 2014).

Definition 2.8. Suppose that $X(u, v)=a(u)+v b(u)$ is a non-developable ruled surface in $E^{3}$ and $a(u)$ is the striction line of $X(u, v)$ under the condition $a^{\prime}(u)=\lambda(u) x(u)+\mu(u) y(u)$. Here, $\{\alpha(u), x(u)=b(u), y(u)\} \quad$ is the spherical Frenet frame of the spherical curve $b(u)$ and the parameter $u$ is the arc length parameter of $b(u)$.

We assume that $\lambda(u) \neq 0$, then $X(u, v)$ is described as pitched ruled surface. (Liu et al., 2014; Yu et al., 2014). 


\section{RESULTS AND DISCUSSION}

Let $X(u, v)=a(u)+v b(u)$ be a non-developable $\quad \mu(u)$ and $k_{g}(u)$. Then, the curvature $\kappa$ and torsion ruled surface with the structure functions $\lambda(u), \quad \tau$ of striction line $a(u)$ are, respectively, given by

$$
\kappa^{2}=\frac{\left(\lambda-k_{g} \mu\right)^{2}\left(\lambda^{2}+\mu^{2}\right)+\left(\lambda^{\prime} \mu-\lambda \mu^{\prime}\right)^{2}}{\left(\lambda^{2}+\mu^{2}\right)^{3}}
$$

and

$\tau=\frac{\left(\lambda-\mu k_{g}\right)\left(\lambda \mu^{\prime \prime}-\lambda^{\prime \prime} \mu+\left(\lambda k_{g}+\mu\right)\left(\lambda-\mu k_{g}\right)\right)+\left(\lambda^{\prime} \mu-\lambda \mu^{\prime}\right)\left(2 \lambda^{\prime}-2 k_{g} \mu^{\prime}-k_{g}{ }^{\prime} \mu\right)}{\left(\lambda-k_{g} \mu\right)^{2}\left(\lambda^{2}+\mu^{2}\right)+\left(\lambda^{\prime} \mu-\lambda \mu^{\prime}\right)^{2}}$

(Liu et al., 2014; Yu et al., 2014).

From “'Eq. 10.” and “Eq. 11.”, we obtain

$$
\begin{aligned}
& \left(\lambda-\mu k_{g}\right)\left(\lambda \mu^{\prime \prime}-\lambda^{\prime \prime} \mu+\left(\lambda k_{g}+\mu\right)\left(\lambda-\mu k_{g}\right)\right)
\end{aligned}
$$

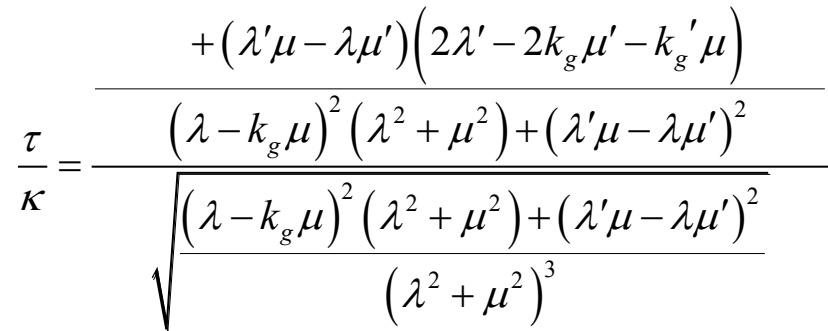

By definition of helix, the last equation should be constant. In this case, if we choose

$$
\frac{\lambda}{\mu}=\text { constant }, \text { we get }
$$

$\lambda^{\prime} \mu-\lambda \mu^{\prime}=0$

and

$$
\lambda \mu^{\prime \prime}-\lambda^{\prime \prime} \mu=0
$$

Then, we have

$$
\begin{aligned}
& \frac{\tau}{\kappa}=\frac{\left(\lambda-\mu k_{g}\right)\left(\lambda k_{g}+\mu\right)\left(\lambda-\mu k_{g}\right)}{\left(\lambda-\mu k_{g}\right)^{2}\left(\lambda^{2}+\mu^{2}\right)} \frac{\left(\lambda^{2}+\mu^{2}\right) \sqrt{\lambda^{2}+\mu^{2}}}{\left(\lambda-\mu k_{g}\right) \sqrt{\left(\lambda^{2}+\mu^{2}\right)}} \\
& \frac{\tau}{\kappa}=\frac{\lambda k_{g}+\mu}{\lambda-k_{g} \mu} .
\end{aligned}
$$


If striction line is helix "Eq. 16." is constant and so, we can write

$\frac{\lambda k_{g}+\mu}{\lambda-k_{g} \mu}=c,(c \in I R)$

and we obtain

$k_{g}=\frac{c \lambda-\mu}{\lambda+c \mu}$.

From here, we can express the following theorem:

Theorem 3.1. Suppose that $X(u, v)=a(u)+v b(u)$ is any non-developable ruled surface in $E^{3}$ and $a(u)$ is the striction line of $X(u, v)$ under the condition $a^{\prime}=\lambda x(u)+\mu y(u)$. $a(u)$ is a helix if and only if $\frac{\lambda}{\mu}=$ constant and $k_{g}=\frac{c \lambda-\mu}{\lambda+c \mu},(c \in R)$.

By the definition of slant helix, "Eq. 3." should be constant. From "Eq. 10." and "Eq.

11.', if we choose $\frac{\lambda}{\mu}=$ constant, we have

$\kappa=\frac{\lambda-k_{g} \mu}{\lambda^{2}+\mu^{2}}$

and

$\tau=\frac{\lambda k_{g}+\mu}{\lambda^{2}+\mu^{2}}$

Then, we obtain

$$
\kappa^{2}+\tau^{2}=\frac{1+k_{g}^{2}}{\lambda^{2}+\mu^{2}}
$$

$\left(\frac{\tau}{\kappa}\right)^{\prime}=\left(\frac{\lambda k_{g}+\mu}{\lambda-k_{g} \mu}\right)^{\prime}=\frac{\left(\lambda^{\prime} k_{g}+\lambda k_{g}{ }^{\prime}+\mu^{\prime}\right)\left(\lambda-k_{g} \mu\right)-\left(\lambda^{\prime}-k_{g}{ }^{\prime} \mu-k_{g} \mu^{\prime}\right)\left(\lambda k_{g}+\mu\right)}{\left(\lambda-k_{g} \mu\right)^{2}}$

$\left(\frac{\tau}{\kappa}\right)^{\prime}=\frac{k_{g}^{\prime}\left(\lambda^{2}+\mu^{2}\right)}{\left(\lambda-k_{g} \mu\right)^{2}}$ 
$\frac{\frac{\left(\lambda-k_{g} \mu\right)^{2}}{\left(\lambda^{2}+\mu^{2}\right)^{2}}}{\left(\frac{1+k_{g}^{2}}{\lambda^{2}+\mu^{2}}\right)^{\frac{3}{2}}} \frac{k_{g}^{\prime}\left(\lambda^{2}+\mu^{2}\right)}{\left(\lambda-k_{g} \mu\right)^{2}}=\frac{k_{g}^{\prime}}{\left(1+k_{g}^{2}\right)^{\frac{3}{2}}}$.

Here, if we choose $\frac{k_{g}{ }^{\prime}}{\left(1+k_{g}^{2}\right)^{\frac{3}{2}}}=c$ and solve this differential equation, we obtain

$\frac{k_{g}}{\sqrt{1+k_{g}^{2}}}=c u+d,(c, d$ constant $)$.

Therefore, we can say the following theorem:

Theorem 3.2. Suppose that $X(u, v)=a(u)+v b(u)$ is a non-developable ruled surface in $E^{3}$ and $a(u)$ is the striction line of $X(u, v)$ under the condition $a^{\prime}(u)=\lambda x(u)+\mu y(u) . \quad a(u)$ is a slant helix if and only if $\frac{\lambda}{\mu}=$ constant and $\frac{k_{g}}{\sqrt{1+k_{g}^{2}}}=c u+d$, where $k_{g}(u), \lambda(u)$ and $\mu(u)$ are structure functions of $X(u, v)$ and $c, d$ are constants.

It is know that a space curve is a Bertrand curve under the condition that $A\left(\tau^{\prime} \kappa-\tau \kappa^{\prime}\right)=\tau^{\prime}$

where $A$ is nonzero a real number. From "Eq. 26.", we can write $\left(\frac{\tau}{\kappa}\right)^{\prime} \kappa^{2} A=\tau^{\prime}$

Here, if we use value in "Eq. 23." instead of $\left(\frac{\tau}{\kappa}\right)^{\prime}$ and consider $\frac{\lambda}{\mu}=$ constant, we get

$$
\frac{k_{g}{ }^{\prime}\left(\lambda^{2}+\mu^{2}\right)}{\left(\lambda-\mu k_{g}\right)^{2}} \frac{\left(\lambda-\mu k_{g}\right)^{2}}{\left(\lambda^{2}+\mu^{2}\right)^{2}} A=\lambda^{\prime} k_{g}+\lambda k_{g}{ }^{\prime}+\mu^{\prime}
$$




$$
\begin{aligned}
& \frac{k_{g}{ }^{\prime}\left(\lambda^{2}+\mu^{2}\right)}{\left(\lambda-\mu k_{g}\right)^{2}} \frac{\left(\lambda-\mu k_{g}\right)^{2}}{\left(\lambda^{2}+\mu^{2}\right)^{2}} A=\lambda^{\prime} k_{g}+\lambda k_{g}{ }^{\prime}+\mu^{\prime} \\
& A=\lambda+\frac{\lambda^{\prime} k_{g}+\mu^{\prime}}{k_{g}{ }^{\prime}},
\end{aligned}
$$

where $u$ is the arc length parameter of $a(u)$. Since $A$ is a non-zero constant, $\lambda$ and $\mu$ are constants. In this case, we can write the following theorem:

Theorem 3.3. Suppose that $X(u, v)=a(u)+v b(u)$ is any non-developable ruled surface in $E^{3}$ and $a(u)$ is the striction line of $X(u, v)$ under the condition $a^{\prime}(u)=\lambda x(u)+\mu y(u) . a(u)$ is a Bertrand curve if and only if $\lambda$ and $\mu$ are constants, where $u$ is arc length parameter of $a(u)$.

$$
\begin{aligned}
& \kappa=c\left(\kappa^{2}+\tau^{2}\right) \\
& c \frac{1+k_{g}^{2}}{\lambda^{2}+\mu^{2}}=\frac{\lambda-k_{g} \mu}{\lambda^{2}+\mu^{2}} \\
& c=\frac{\lambda-k_{g} \mu}{1+k_{g}^{2}},
\end{aligned}
$$

where $c \neq 0, c \in I R$.

Hence, we can give the following theorem:

Theorem 3.4. Suppose that $X(u, v)=a(u)+v b(u)$ is a non-developable ruled surface in $E^{3}$ and
Corollary 3.1. The striction line $a(u)$ of $X(u, v)$ is a Bertrand curve if and only if $A=\lambda$.

Proof: If $\lambda$ and $\mu$ are considered constants in “Eq. 29.", it can be easily obtained $A=\lambda$.

It is just known that a space curve in $E^{3}$ is a Mannheim curve if "Eq. 5." is provided. If we suppose that $\frac{\lambda}{\mu}$ is constant and use "Eq. 19.", “Eq. 20.” and “Eq. 21.”, we obtain

$a(u)$ is the striction line of $X(u, v)$ under the condition 
$a^{\prime}(u)=\lambda x(u)+\mu y(u) . a(u)$ is a Mannheim curve if and only if $\frac{\lambda}{\mu}=$ constant and $c=\frac{\lambda-k_{g} \mu}{1+k_{g}^{2}}$, where $k_{g}(u), \lambda(u)$ and $\mu(u)$ are structure functions of $X(u, v)$ and $c$ is a non-zero constant.

\section{CONCLUSION}

In this paper, firstly, we gave definitions of helix, slant helix, Bertrand and Mannheim curves. Then, non-developable ruled surfaces and striction lines of non-developable ruled

\section{REFERENCES}

Izumiya S, Takeuchi N, 2003. Special Curves and Ruled Surfaces. Contributions to Algebra and Geometry, 44: 203-212.

Kuhnel W, 2006. Differential geometry, Curves-Surfaces-Manifolds. Second Edition, American Mathematical Society, USA. 380p.

Liu H, Wang F, 2008. Mannheim partner curves in 3-space. Journal of Geometry, 88: 120-126.

Liu H, Yu Y, Jung SD, 2014. Invariants of non-developable ruled surfaces in Euclidean 3-space. Contrib. Algebra Geom., 55: 189-199. surfaces was defined by means of structure functions. Finally, we showed that under certain conditions, striction lines of nondevelopable ruled surfaces are helix, slant helix, Bertrand or Mannheim curve.

Yaylı Y, Saraçoğlu S, 2012. Different Approaches To Ruled Surfaces. SDU Journal of Science, 7 (1): 56-68

Yoon DW, 2007. On Non-Developable Ruled Surfaces in Euclidean 3- Spaces. Indian J. pure appl. Math., 38(4): 281-290.

Yu Y, Liu H, Jung SD, 2014. Structure and characterization of ruled surfaces in Euclidean 3-space. Applied Mathematics and Computation, 233: 252259. 